\title{
Research Article \\ Generalized Difference Spaces of Non-Absolute Type of Convergent and Null Sequences
}

\author{
Abdulcabbar Sönmez ${ }^{1}$ and Feyzi Başar ${ }^{2}$ \\ ${ }^{1}$ Department of Mathematics, Faculty of Sciences, Erciyes University, Melikgazi, 38039 Kayseri, Turkey \\ 2 Department of Mathematics, Faculty of Art and Sciences, Fatih University, The Hadımköy Campus, \\ Büyükçekmece, 34500 İstanbul, Turkey
}

Correspondence should be addressed to Abdulcabbar Sönmez, sonmez@erciyes.edu.tr

Received 17 February 2012; Accepted 10 October 2012

Academic Editor: Stevo Stevic

Copyright @ 2012 A. Sönmez and F. Başar. This is an open access article distributed under the Creative Commons Attribution License, which permits unrestricted use, distribution, and reproduction in any medium, provided the original work is properly cited.

The aim of the present paper is to introduce the spaces $c_{0}^{\lambda}(B)$ and $c^{\lambda}(B)$ of generalized difference sequences which generalize the paper due to Mursaleen and Noman (2010). These spaces are the $B K$-spaces of non-absolute type and norm isomorphic to the spaces $c_{0}$ and $c$, respectively. Furthermore, we derive some inclusion relations determine the $\alpha-, \beta-$, and $\gamma$-duals of those spaces, and construct their Schauder bases. Finally, we characterize some matrix classes from the spaces $c_{0}^{\lambda}(B)$, and $c^{\lambda}(B)$ to the spaces $\ell_{p}, c_{0}$, and $c$.

\section{Introduction}

By $\omega$, we denote the space of all complex valued sequences. Any vector subspace of $\omega$ is called a sequence space. A sequence space $E$ with a linear topology is called a $K$-space provided each of the maps $p_{i}: E \rightarrow \mathbb{C}$ defined by $p_{i}(x)=x_{i}$ is continuous for all $i \in \mathbb{N}$, where $\mathbb{C}$ denotes the complex field and $\mathbb{N}=\{0,1,2, \ldots\}$. A $K$-space is called an $F K$-space provided $E$ is a complete linear metric space. An $F K$-space whose topology is normable is called a $B K$-space (see [1, pages 272-273]) which contains $\phi$, the set of all finitely nonzero sequences. We write $\ell_{\infty}, c$ and $c_{0}$ for the classical sequence spaces of all bounded, convergent, and null sequences, respectively, which are $B K$-spaces with the usual sup-norm defined by $\|x\|_{\infty}=$ $\sup \left|x_{k}\right|$, where, here and in the sequel, the supremum is taken over all $k \in \mathbb{N}$. Also by $\ell_{1}$ and $\ell_{p}$, we denote the spaces of all absolutely and $p$-absolutely convergent series, respectively, which are $B K$-spaces with the usual norm defined by $\|x\|_{p}=\left(\sum_{k}\left|x_{k}\right|^{p}\right)^{1 / p}$, where $1 \leq p<$ $\infty$. For simplicity in notation, here and in what follows, the summation without limits runs 
from 0 to $\infty$. Also by bs and cs, we denote the spaces of all bounded and convergent series, respectively.

Let $X$ and $Y$ be two sequence spaces, and let $A=\left(a_{n k}\right)$ be an infinite matrix of complex numbers $a_{n k}$, where $n, k \in \mathbb{N}$. Then, we say that $A$ defines a matrix mapping from $X$ into $Y$ and we denote it by writing $A: X \rightarrow Y$, if for every sequence $x=\left(x_{k}\right) \in X$ the sequence $A x=\left\{A_{n}(x)\right\}, A$-transform of $x$, exists and is in $Y$, where

$$
A_{n}(x):=\sum_{k} a_{n k} x_{k}, \quad \forall n \in \mathbb{N}
$$

By $(X: Y)$, we denote the class of all infinite matrices $A=\left(a_{n k}\right)$ such that $A: X \rightarrow Y$. Thus $A \in(X: Y)$ if and only if the series on the right side of (1.1) converges for each $n \in \mathbb{N}$ and every $x \in X$, and $A x \in Y$ for all $x \in X$. A sequence $x \in \omega$ is said to be $A$-summable to $l$ if $A x$ converges to $l$, which is called the $A$-limit of $x$.

The domain $X_{A}$ of an infinite matrix $A$ in a sequence space $X$ is defined by

$$
X_{A}:=\left\{x=\left(x_{k}\right) \in \omega: A x \in X\right\}
$$

We denote the collection of all finite subsets of $\mathbb{N}$ by $\mathcal{F}$. Also, we write $e^{(k)}$ for the sequence whose only nonzero term is a 1 in the $k$ th place for each $k \in \mathbb{N}$.

The approach of constructing a new sequence space by means of the matrix domain of a particular limitation method has recently been employed by several authors, for example, [2-14]. They introduced the sequence spaces $\left(\ell_{\infty}\right)_{N_{q}}$ and $c_{N_{q}}$ in [14], $\left(\ell_{p}\right)_{C_{1}}=X_{p}$ and $\left(\ell_{\infty}\right)_{C_{1}}=$ $X_{\infty}$ in [10], $\mu_{G}=Z(u, v ; \mu)$ in [9], $\left(\ell_{\infty}\right)_{R^{t}}=r_{\infty}^{t}, c_{R^{t}}=r_{c}^{t}$ and $\left(c_{0}\right)_{R^{t}}=r_{0}^{t}$ in [8], $\left(\ell_{p}\right)_{R^{t}}=r_{p}^{t}$ in [2], $\left(c_{0}\right)_{E^{t}}=e_{0}^{r}$ and $c_{E^{r}}=e_{c}^{r}$ in [3], $\left(\ell_{p}\right)_{E^{r}}=e_{p}^{r}$ and $\left(\ell_{\infty}\right)_{E^{r}}=e_{\infty}^{r}$ in [4], $\left(c_{0}\right)_{A^{r}}=a_{0}^{r}$ and $c_{A^{r}}=a_{c}^{r}$ in [5], $\left[c_{0}(u, p)\right]_{A^{r}}=a_{0}^{r}(u, p)$ and $[c(u, p)]_{A^{r}}=a_{c}^{r}(u, p)$ in [6], $\left(\ell_{p}\right)_{A^{r}}=a_{p}^{r}$ and $\left(\ell_{\infty}\right)_{A^{r}}=a_{\infty}^{r}$ in [7] and $\left(c_{0}\right)_{C_{1}}=\widetilde{c}_{0}$ and $c_{C_{1}}=\tilde{c}$ in [11], $v_{B(r, s, t)}=\mathcal{v}(B)$ in [12], and $f_{B(r, s, t)}=f(B)$ in [13]; where, $N_{q}, C_{1}, R^{t}$, and $E^{r}$ denote the Nörlund, Cesáro, Riesz, and Euler means, respectively, $A^{r}, G$, and $B(r, s, t)$ are, respectively, defined in $[5,9,12], \mu \in\left\{c_{0}, c, \ell_{p}\right\}, v \in\left\{\ell_{\infty}, c, c_{0}, \ell_{p}\right\}$ and $1 \leq p<\infty$. Also $c_{0}(u, p)$ and $c(u, p)$ denote the sequence spaces generated from the Maddox's spaces $c_{0}(p)$ and $c(p)$ by Başarir [15]. In the present paper, following [2-14], we introduce the difference sequence spaces $c_{0}^{\lambda}(B)$ and $c^{\lambda}(B)$ of non-absolute type and derive some related results. We also establish some inclusion relations. Furthermore, we determine the $\alpha^{-}, \beta-$, and $\gamma$-duals of those spaces and construct their bases. Finally, we characterize some classes of infinite matrices concerning the spaces $c_{0}^{\lambda}(B)$ and $c^{\lambda}(B)$.

The rest of this paper is organized, as follows.

In Section 2, the $B K$-spaces $c_{0}^{\lambda}(B)$ and $c^{\lambda}(B)$ of generalized difference sequences are introduced. Section 3 is devoted to inclusion relations concerning with the spaces $c_{0}^{\lambda}(B)$ and $c^{\lambda}(B)$. In Sections 4 and 5 , the Schauder bases of the spaces $c_{0}^{\lambda}(B)$ and $c^{\lambda}(B)$ are given and the $\alpha-, \beta$-, and $\gamma$-duals of the generalized difference sequence spaces $c_{0}^{\lambda}(B)$ and $c^{\lambda}(B)$ of non-absolute type are determined, respectively. In Section 6 , the classes $\left(c^{\lambda}(B): \ell_{p}\right)$, $\left(c_{0}^{\lambda}(B): \ell_{p}\right),\left(c^{\lambda}(B): c\right),\left(c^{\lambda}(B): c_{0}\right),\left(c_{0}^{\lambda}(B): c\right)$, and $\left(c_{0}^{\lambda}(B): c_{0}\right)$ of matrix transformations are characterized, where $1 \leq p \leq \infty$. Also, by means of a given basic lemma, the characterizations of some other classes involving the Euler, difference, Riesz, and Cesàro sequence spaces are derived. In the final section of the paper, we note the significance of the present results in the literature related with difference sequence spaces and record some further suggestions. 


\section{The Difference Sequence Spaces $c_{0}^{\lambda}(B)$ and $c^{\lambda}(B)$ of Non-Absolute Type}

The difference sequence spaces have been studied by several authors in different ways (see e.g. $[12,16-21])$. In the present section, we introduce the spaces $c_{0}^{\lambda}(\Delta)$, and $c^{\lambda}(\Delta)$, and show that these spaces are $B K$-spaces of non-absolute type which are norm isomorphic to the spaces $c_{0}$ and $c$, respectively.

We assume throughout that $\lambda=\left(\lambda_{k}\right)_{k=0}^{\infty}$ is a strictly increasing sequence of positive reals tending to $\infty$, that is,

$$
0<\lambda_{0}<\lambda_{1}<\cdots, \quad \lim _{k \rightarrow \infty} \lambda_{k}=\infty
$$

Recently, Mursaleen and Noman [22] studied the sequence spaces $c_{0}^{\lambda}$ and $c^{\lambda}$ of nonabsolute type, and later they introduced the difference sequence spaces $c_{0}^{\mathcal{\lambda}}(\Delta)$ and $c^{\mathcal{\lambda}}(\Delta)$ in [21] of non-absolute type as follows:

$$
\begin{gathered}
c_{0}^{\lambda}(\Delta)=\left\{x=\left(x_{k}\right) \in \omega: \lim _{n \rightarrow \infty} \frac{1}{\lambda_{n}} \sum_{k=0}^{n}\left(\lambda_{k}-\lambda_{k-1}\right)\left(x_{k}-x_{k-1}\right)=0\right\}, \\
c^{\lambda}(\Delta)=\left\{x=\left(x_{k}\right) \in \omega: \lim _{n \rightarrow \infty} \frac{1}{\lambda_{n}} \sum_{k=0}^{n}\left(\lambda_{k}-\lambda_{k-1}\right)\left(x_{k}-x_{k-1}\right) \text { exists }\right\} .
\end{gathered}
$$

Here and after, we use the convention that any term with a negative subscript is equal to zero, for example, $\lambda_{-1}=0$ and $x_{-1}=0$. With the notation of (1.2) we can redefine the spaces $c_{0}^{\lambda}(\Delta)$ and $c^{\curlywedge}(\Delta)$ by

$$
c_{0}^{\lambda}(\Delta)=\left(c_{0}^{\lambda}\right)_{\Delta^{\prime}} \quad c^{\lambda}(\Delta)=\left(c^{\curlywedge}\right)_{\Delta^{\prime}}
$$

where $\Delta$ denotes the band matrix representing the difference operator, that is, $\Delta x=\left(x_{k}-\right.$ $\left.x_{k-1}\right) \in \omega$ for $x=\left(x_{k}\right) \in \omega$.

Let $r$ and $s$ be nonzero real numbers and define the generalized difference matrix $B(r, s)=\left\{b_{n k}(r, s)\right\}$ by

$$
b_{n k}(r, s):= \begin{cases}r, & k=n \\ s, & k=n-1 \\ 0, & \text { otherwise }\end{cases}
$$

for all $k, n \in \mathbb{N}$. The $B(r, s)$-transform of a sequence $x=\left(x_{k}\right)$ is

$$
B(r, s)_{k}(x)=r x_{k}+s x_{k-1}, \quad \forall k \in \mathbb{N} .
$$

We note that the matrix $B(r, s)$ can be reduced to the difference matrices $\Delta$ in case $r=1$ and $s=-1$. So, the results related to the matrix domain of the matrix $B(r, s)$ are more general and more comprehensive than the consequences of the matrices domain of $\Delta$ and include them. 
Now, following Başar and Altay [18] and Aydın and Başar [17], we proceed slightly differently to Kizmaz [19] and the other authors following him and employ a technique of obtaining a new sequence space by means of the matrix domain of a triangle limitation method.

We thus introduce the difference sequence spaces $c_{0}^{\lambda}(B)$ and $c^{\lambda}(B)$, which are the generalization of the spaces $c_{0}^{\lambda}(\Delta)$ and $c^{\lambda}(\Delta)$ introduced by Mursaleen and Noman [21], as follows:

$$
\begin{gathered}
c_{0}^{\lambda}(B)=\left\{x=\left(x_{k}\right) \in \omega: \lim _{n \rightarrow \infty} \frac{1}{\lambda_{n}} \sum_{k=0}^{n}\left(\lambda_{k}-\lambda_{k-1}\right)\left(r x_{k}+s x_{k-1}\right)=0\right\}, \\
c^{\lambda}(B)=\left\{x=\left(x_{k}\right) \in \omega: \lim _{n \rightarrow \infty} \frac{1}{\lambda_{n}} \sum_{k=0}^{n}\left(\lambda_{k}-\lambda_{k-1}\right)\left(r x_{k}+s x_{k-1}\right) \text { exists }\right\} .
\end{gathered}
$$

With the notation of (1.2), we can redefine the spaces $c_{0}^{\lambda}(B)$ and $c^{\lambda}(B)$ as

$$
c_{0}^{\lambda}(B)=\left(c_{0}^{\lambda}\right)_{B^{\prime}} \quad c^{\lambda}(B)=\left(c^{\curlywedge}\right)_{B^{\prime}}
$$

where $B$ denotes the generalized difference matrix $B(r, s)=\left\{b_{n k}(r, s)\right\}$ defined by (2.4).

It is immediate by $(2.7)$ that the sets $c_{0}^{\lambda}(B)$ and $c^{\lambda}(B)$ are linear spaces with coordinatewise addition and scalar multiplication, that is, $c_{0}^{\lambda}(B)$ and $c^{\lambda}(B)$ are the sequence spaces of generalized differences.

On the other hand, we define the triangle matrix $\widehat{\Lambda}=\left(\widehat{\lambda}_{n k}\right)$ by

$$
\hat{\lambda}_{n k}:= \begin{cases}\frac{r\left(\lambda_{k}-\lambda_{k-1}\right)+s\left(\lambda_{k+1}-\lambda_{k}\right)}{\lambda_{n}}, & k<n, \\ r \frac{\left(\lambda_{n}-\lambda_{n-1}\right)}{\lambda_{n}}, & k=n, \\ 0, & k>n\end{cases}
$$

for all $n, k \in \mathbb{N}$. With a direct calculation we derive the equality

$$
\widehat{\Lambda}_{n}(x)=\frac{1}{\lambda_{n}} \sum_{k=0}^{n}\left(\lambda_{k}-\lambda_{k-1}\right)\left(r x_{k}+s x_{k-1}\right), \quad \forall n \in \mathbb{N}
$$

and every $x=\left(x_{k}\right) \in \omega$ which leads us together with (1.2) to the fact that

$$
c_{0}^{\lambda}(B)=\left(c_{0}\right)_{\widehat{\Lambda}^{\prime}}, \quad c^{\lambda}(B)=c_{\widehat{\Lambda}} .
$$

Further, for any sequence $x=\left(x_{k}\right)$ we define the sequence $y(\lambda)=\left\{y_{k}(\lambda)\right\}$ which will be frequently used as the $\widehat{\Lambda}$-transform of $x$, that is, $y(\lambda)=\widehat{\Lambda}(x)$ and so we have

$$
y_{k}(\lambda)=\sum_{j=0}^{k-1} \frac{r\left(\lambda_{j}-\lambda_{j-1}\right)+s\left(\lambda_{j+1}-\lambda j\right)}{\lambda_{k}} x_{j}+r \frac{\lambda_{k}-\lambda_{k-1}}{\lambda_{k}} x_{k}, \quad \forall k \in \mathbb{N},
$$


Where, here and in what follows, the summation running from 0 to $k-1$ is equal to zero when $k=0$.

Moreover, it is clear by (2.9) that the relation (2.11) can be written as follows:

$$
y_{k}(\lambda)=\frac{1}{\lambda_{k}} \sum_{j=0}^{k}\left(\lambda_{j}-\lambda_{j-1}\right)\left(r x_{j}+s x_{j-1}\right), \quad \forall k \in \mathbb{N}
$$

We assume throughout that the sequences $x=\left(x_{k}\right)$ and $y=\left(y_{k}\right)$ are connected by the relation (2.11).

Now, we may begin with the following theorem which is essential in the text.

Theorem 2.1. The difference sequence spaces $c_{0}^{\lambda}(B)$ and $c^{\lambda}(B)$ are $B K$-spaces with the norm $\|x\|_{\mathcal{C}_{0}^{\lambda}(B)}=\|x\|_{c^{\lambda}(B)}=\|\widehat{\Lambda}(x)\|_{\infty}$, that is,

$$
\|x\|_{\mathcal{C}_{0}^{\lambda}(B)}=\|x\|_{\mathcal{C}^{\lambda}(B)}=\sup _{n \in \mathbb{N}}\left|\widehat{\Lambda}_{n}(x)\right|
$$

Proof. Since (2.10) holds and $c_{0}$ and $c$ are $B K$-spaces with respect to their natural norms (see [23, pages 217-218]) and the matrix $\widehat{\Lambda}$ is a triangle, Theorem 4.3.12 of Wilansky [24, page 63] gives the fact that $c_{0}^{\lambda}(B)$ and $c^{\lambda}(B)$ are $B K$-spaces with the given norms. This completes the proof.

Remark 2.2. One can easily check that the absolute property does not hold on the spaces $c_{0}^{\lambda}(B)$ and $c^{\lambda}(B)$, that is, $\|x\|_{c_{0}^{\lambda}(B)} \neq\||x|\|_{c_{0}^{\lambda}(B)}$ and $\|x\|_{c^{\lambda}(B)} \neq\|x \mid\|_{c^{\lambda}(B)}$ for at least one sequence in the spaces $c_{0}^{\lambda}(B)$ and $c^{\lambda}(B)$, and this shows that $c_{0}^{\lambda}(B)$ and $c^{\lambda}(B)$ are the sequence spaces of nonabsolute type, where $|x|=\left(\left|x_{k}\right|\right)$.

Now, we give the final theorem of this section.

Theorem 2.3. The sequence spaces $c_{0}^{\lambda}(B)$ and $c^{\lambda}(B)$ of non-absolute type are norm isomorphic to the spaces $c_{0}$ and $c$, respectively, that is, $c_{0}^{\mathcal{\lambda}}(B) \cong c_{0}$ and $c^{\mathcal{l}}(B) \cong c$.

Proof. To prove this, we should show the existence of a linear bijection between the spaces $c_{0}^{\lambda}(B)$ and $c_{0}$. Consider the transformation $T$ defined, with the notation of (2.11), from $c_{0}^{\lambda}(B)$ to $c_{0}$ by $x \mapsto y(\lambda)$. Then, $T x=y(\lambda)=\widehat{\Lambda}(x) \in c_{0}$ for every $x \in c_{0}^{\lambda}(B)$ and the linearity of $T$ is clear. Further, it is trivial that $x=\theta$ whenever $T x=\theta$ and hence $T$ is injective.

Furthermore, let $y=\left(y_{k}\right) \in c_{0}$ and define the sequence $x=\left\{x_{k}(\lambda)\right\}$ by

$$
x_{k}(\lambda):=\frac{1}{r} \sum_{j=0}^{k}\left(\frac{-s}{r}\right)^{k-j} \sum_{i=j-1}^{j}(-1)^{j-i} \frac{\lambda_{i}}{\lambda_{j}-\lambda_{j-1}} y_{i}, \quad \forall k \in \mathbb{N} .
$$

Then, we obtain

$$
r x_{k}(\lambda)+s x_{k-1}(\lambda)=\sum_{i=k-1}^{k}(-1)^{k-i} \frac{\lambda_{i}}{\lambda_{k}-\lambda_{k-1}} y_{i}, \quad \forall k \in \mathbb{N} .
$$


Hence, for every $n \in \mathbb{N}$, we get by (2.9)

$$
\begin{aligned}
\widehat{\Lambda}_{n}(x) & =\frac{1}{\lambda_{n}} \sum_{k=0}^{n}\left(\lambda_{k}-\lambda_{k-1}\right)\left(r x_{k}+s x_{k-1}\right) \\
& =\frac{1}{\lambda_{n}} \sum_{k=0}^{n} \sum_{i=k-1}^{k}(-1)^{k-i} \lambda_{i} y_{i} \\
& =y_{n} .
\end{aligned}
$$

This shows that $\widehat{\Lambda}(x)=y$ and since $y \in c_{0}$, we conclude that $\widehat{\Lambda}(x) \in c_{0}$. Thus, we deduce that $x \in c_{0}^{\lambda}(B)$ and $T x=y$. Hence $T$ is surjective.

Moreover, one can easily see for every $x \in c_{0}^{\curlywedge}(B)$ that

$$
\|T x\|_{\infty}=\|y(\lambda)\|_{\infty}=\|\hat{\Lambda}(x)\|_{\infty}=\|x\|_{\mathcal{C}_{0}^{\lambda}(B)}
$$

which means that $T$ is norm preserving. Consequently $T$ is a linear bijection which show that the spaces $c_{0}^{\lambda}(B)$ and $c_{0}$ are linearly isomorphic.

It is clear that if the spaces $c_{0}^{\lambda}(B)$ and $c_{0}$ are replaced by the spaces $c^{\lambda}(B)$ and $c$, respectively, then we obtain the fact that $c^{\lambda}(B) \cong c$. This completes the proof.

\section{The Inclusion Relations}

In the present section, we establish some inclusion relations concerning with the spaces $c_{0}^{\lambda}(B)$ and $c^{\lambda}(B)$. We may begin with the following theorem.

Theorem 3.1. The inclusion $c_{0}^{\mathcal{l}}(B) \subset c^{\curlywedge}(B)$ strictly holds.

Proof. It is obvious that the inclusion $c_{0}^{\lambda}(B) \subset c^{\lambda}(B)$ holds. Further to show that this inclusion is strict, consider the sequence $x=\left(x_{k}\right)$ defined by $x_{k}=\sum_{j=0}^{k}(-s / r)^{j} / r$ for all $k \in \mathbb{N}$. Then, we obtain by (2.9) that

$$
\widehat{\Lambda}_{n}(x)=\frac{1}{\lambda_{n}} \sum_{k=0}^{n}\left(\lambda_{k}-\lambda_{k-1}\right) \quad \forall n \in \mathbb{N}
$$

which shows that $\widehat{\Lambda}(x)=e$ and hence $\widehat{\Lambda}(x) \in c \backslash c_{0}$, where $e=(1,1,1, \ldots)$. Thus, the sequence $x$ is in $c^{\lambda}(B)$ but not in $c_{0}^{\lambda}(B)$. Hence, the inclusion $c_{0}^{\lambda}(B) \subset c^{\lambda}(B)$ is strict and this completes the proof.

Theorem 3.2. If $s+r=0$, then the inclusion $c \subset c_{0}^{\lambda}(B)$ strictly holds.

Proof. Suppose that $s+r=0$ and $x \in c$. Then $B(r, s) x=\left(r x_{k}+s x_{k-1}\right) \in c_{0}$ and hence $B(r, s) x \in$ $c_{0}^{\lambda}$, since the inclusion $c_{0} \subset c_{0}^{\lambda}$. This shows that $x \in c_{0}^{\lambda}(B)$. Consequently, the inclusion $c \subset$ $c_{0}^{\lambda}(B)$ holds. Further consider the sequence $y=\left(y_{k}\right)$ defined by $y_{k}=\sqrt{k+1}$ for all $k \in \mathbb{N}$. Then, it is trivial that $y \notin c$. On the other hand, it can easily seen that $B(r, s) y \in c_{0}$. Hence, 
$B(r, s) y \in c_{0}^{\lambda}$ which means that $y \in c_{0}^{\lambda}(B)$. Thus, the sequence $y$ is in $c_{0}^{\lambda}(B)$ but not in $c$. We therefore deduce that the inclusion $c \subset c_{0}^{\lambda}(B)$ is strict. This completes the proof.

On the other hand, we recall that if $A \in(c: c)$ and $B \in(c: c)$, then $A B \in(c: c)$, namely, $\widehat{\Lambda}=\left(\widehat{\lambda}_{n k}\right)$ is stronger than the ordinary convergence, hence we have the following

Corollary 3.3. The inclusions $c_{0} \subset c_{0}^{\lambda}(B)$ and $c \subset c^{\lambda}(B)$ strictly hold.

Further, it is obvious that the sequence $y$, defined in the proof of Theorem 3.2, is in $c_{0}^{\lambda}(B)$ but not in $\ell_{\infty}$. This leads us to the following result.

Corollary 3.4. Although the spaces $\ell_{\infty}$ and $c_{0}^{\lambda}(B)$ overlap, the space $\ell_{\infty}$ does not include the space $c_{0}^{\lambda}(B)$.

Now, to prove the next theorem, we need the following lemma [24, page 4].

Lemma 3.5. $A \in\left(\ell_{\infty}: c_{0}\right)$ if and only if $\lim _{n \rightarrow \infty} \sum_{k}\left|a_{n k}\right|=0$.

Theorem 3.6. The inclusion $\ell_{\infty} \subset c_{0}^{\lambda}(B)$ strictly holds if and only if $z \in c_{0}^{\lambda}$, where the sequence $z=\left(z_{k}\right)$ is defined by

$$
z_{k}=\left|\frac{r\left(\lambda_{k}-\lambda_{k-1}\right)+s\left(\lambda_{k+1}-\lambda_{k}\right)}{\lambda_{k}-\lambda_{k-1}}\right|, \quad \forall k \in \mathbb{N}
$$

Proof. Suppose that the inclusion $\ell_{\infty} \subset c_{0}^{\lambda}(B)$ holds. Then we obtain that $\widehat{\Lambda}(x) \in c_{0}$ for every $x \in \ell_{\infty}$ and hence the matrix $\widehat{\Lambda}=\left(\widehat{\lambda}_{n k}\right)$ is in the class $\left(\ell_{\infty}: c_{0}\right)$. Thus it follows by Lemma 3.5 that

$$
\lim _{n \rightarrow \infty} \sum_{k}\left|\hat{\lambda}_{n k}\right|=0
$$

Now, by taking into account the definition of the matrix $\widehat{\Lambda}=\left(\widehat{\lambda}_{n k}\right)$ given by $(2.8)$, we have for every $n \in \mathbb{N}$ that

$$
\sum_{k}\left|\widehat{\lambda}_{n k}\right|=\frac{1}{\lambda_{n}} \sum_{k=0}^{n-1}\left|r\left(\lambda_{k}-\lambda_{k-1}\right)+s\left(\lambda_{k+1}-\lambda_{k}\right)\right|+|r| \frac{\lambda_{n}-\lambda_{n-1}}{\lambda_{n}} .
$$

Thus, the condition (3.3) implies both

$$
\begin{gathered}
\lim _{n \rightarrow \infty}|r| \frac{\lambda_{n}-\lambda_{n-1}}{\lambda_{n}}=0 \\
\lim _{n \rightarrow \infty} \frac{1}{\lambda_{n}} \sum_{k=0}^{n-1}\left|r\left(\lambda_{k}-\lambda_{k-1}\right)+s\left(\lambda_{k+1}-\lambda_{k}\right)\right|=0 .
\end{gathered}
$$


Now we have for every $n \geq 1$ that

$$
\frac{1}{\lambda_{n}} \sum_{k=0}^{n-1}\left|r\left(\lambda_{k}-\lambda_{k-1}\right)+s\left(\lambda_{k+1}-\lambda_{k}\right)\right|=\frac{\lambda_{n-1}}{\lambda_{n}}\left[\frac{1}{\lambda_{n-1}} \sum_{k=0}^{n-1}\left(\lambda_{k}-\lambda_{k-1}\right) z_{k}\right]
$$

and since $\lim _{n \rightarrow \infty}\left(\lambda_{n-1} / \lambda_{n}\right)=1$ by (3.5), we obtain by (3.6) that

$$
\lim _{n \rightarrow \infty} \frac{1}{\lambda_{n-1}} \sum_{k=0}^{n-1}\left(\lambda_{k}-\lambda_{k-1}\right) z_{k}=0
$$

which shows that $z=\left(z_{k}\right) \in c_{0}^{\lambda}$.

Conversely, suppose that $z=\left(z_{k}\right) \in c_{0}^{\lambda}$. Then we have (3.8). Further, for every $n \geq 1$, we derive that

$$
\begin{aligned}
\frac{1}{\lambda_{n}} \sum_{k=0}^{n-1}\left|r\left(\lambda_{k}-\lambda_{k-1}\right)+s\left(\lambda_{k+1}-\lambda_{k}\right)\right| & =\frac{1}{\lambda_{n}} \sum_{k=0}^{n-1}\left(\lambda_{k}-\lambda_{k-1}\right) z_{k} \\
& \leq \frac{1}{\lambda_{n-1}} \sum_{k=0}^{n-1}\left(\lambda_{k}-\lambda_{k-1}\right) z_{k}
\end{aligned}
$$

Then, (3.9) and (3.8) together imply that (3.6) holds. On the other hand, we have for every $n \geq 1$ that

$$
\begin{aligned}
\left|\frac{r \lambda_{n-1}+s\left(\lambda_{n}-\lambda_{0}\right)}{\lambda_{n}}\right| & =\left|\frac{1}{\lambda_{n}} \sum_{k=0}^{n-1} r\left(\lambda_{k}-\lambda_{k-1}\right)+s\left(\lambda_{k+1}-\lambda_{k}\right)\right| \\
& \leq \frac{1}{\lambda_{n}} \sum_{k=0}^{n-1}\left|r\left(\lambda_{k}-\lambda_{k-1}\right)+s\left(\lambda_{k+1}-\lambda_{k}\right)\right| .
\end{aligned}
$$

Therefore, it follows by (3.6) that $\lim _{n \rightarrow \infty}\left[r \lambda_{n-1}+s\left(\lambda_{n}-\lambda_{0}\right)\right] / \lambda_{n}=0$. Particularly, if we take $r=1$ and $s=-1$, then we have $\lim _{n \rightarrow \infty}\left[\lambda_{n}-\lambda_{n-1}-\lambda_{0}\right] / \lambda_{n}=0$ which shows that (3.5) holds. Thus, we deduce by the relation (3.4) that (3.3) holds. This leads us with Lemma 3.5 to the consequence that $\widehat{\Lambda} \in\left(\ell_{\infty}: c_{0}\right)$. Hence, the inclusion $\ell_{\infty} \subset c_{0}^{\lambda}(B)$ holds and is strict by Corollary 3.4. This completes the proof.

\section{The Bases for the Spaces $c_{0}^{\lambda}(B)$ and $c^{\lambda}(B)$}

In the present section, we give two sequences of the points of the spaces $c_{0}^{\lambda}(B)$ and $c^{\lambda}(B)$ which form the bases for those spaces.

If a normed sequence space $X$ contains a sequence $\left(b_{n}\right)$ with the property that for every $x \in X$ there is a unique sequence of scalars $\left(\alpha_{n}\right)$ such that

$$
\lim _{n \rightarrow \infty}\left\|x-\left(\alpha_{0} b_{0}+\alpha_{1} b_{1}+\cdots+\alpha_{n} b_{n}\right)\right\|=0
$$


then $\left(b_{n}\right)$ is called a Schauder basis (or briefly basis) for X. The series $\sum_{k} \alpha_{k} b_{k}$ which has the sum $x$ is then called the expansion of $x$ with respect to $\left(b_{n}\right)$ and is written as $x=\sum_{k} \alpha_{k} b_{k}$.

Now, since the transformation $T$ defined from $c_{0}^{\mathcal{l}}(B)$ to $c_{0}$ in the proof of Theorem 2.3 is an isomorphism, the inverse image of the basis $\left\{e^{(k)}\right\}_{k=0}^{\infty}$ of the space $c_{0}$ is the basis for the new space $c_{0}^{\lambda}(B)$. Therefore, we have the following.

Theorem 4.1. Let $\alpha_{k}(\lambda)=\widehat{\Lambda}_{k}(x)$ for all $k \in \mathbb{N}$ and $l=\lim _{k \rightarrow \infty} \widehat{\Lambda}_{k}(x)$. Define the sequence $b^{(k)}(\lambda)=$ $\left\{b_{n}^{(k)}(\lambda)\right\}_{k=0}^{\infty}$ for every fixed $k \in \mathbb{N}$ by

$$
b_{n}^{(k)}(\lambda):= \begin{cases}\left(\frac{-s}{r}\right)^{n-k}\left[\frac{\lambda_{k}}{r\left(\lambda_{k}-\lambda_{k-1}\right)}+\frac{\lambda_{k}}{s\left(\lambda_{k+1}-\lambda_{k}\right)}\right], & k<n, \\ \frac{1}{r} \frac{\lambda_{k}}{\left(\lambda_{k}-\lambda_{k-1}\right)}, & k=n, \\ 0, & k>n .\end{cases}
$$

Then, the following statements hold.

(a) The sequence $\left\{b^{(k)}(\lambda)\right\}_{k=0}^{\infty}$ is a basis for the space $c_{0}^{\lambda}(B)$ and any $x \in c_{0}^{\lambda}(B)$ has a unique representation of the form $x=\sum_{k} \alpha_{k}(\lambda) b^{(k)}(\lambda)$.

(b) The sequence $\left\{b, b^{(0)}(\lambda), b^{(1)}(\lambda), \ldots\right\}$ is a basis for the space $c^{\lambda}(B)$ and any $x \in c^{\lambda}(B)$ has a unique representation of the form $x=l b+\sum_{k}\left[\alpha_{k}(\lambda)-l\right] b^{(k)}(\lambda)$, where $b=\left(b_{k}\right)=$ $\left\{\sum_{j=0}^{k}(-s / r)^{j} / r\right\}_{k=0}^{\infty}$.

Finally, it easily follows from Theorem 2.1 that $c_{0}^{\lambda}(B)$ and $c^{\lambda}(B)$ are the Banach spaces with their natural norms. Then by Theorem 4.1 we obtain the following.

Corollary 4.2. The difference sequence spaces $c_{0}^{\lambda}(B)$ and $c^{\lambda}(B)$ are seprable.

\section{The $\alpha-, \beta-$, and $\gamma$-Duals of the Spaces $c_{0}^{\lambda}(B)$ and $c^{\lambda}(B)$}

In this section, we state and prove the theorems determining the $\alpha-, \beta-$, and $\gamma$-duals of the generalized difference sequence spaces $c_{0}^{\lambda}(B)$ and $c^{\lambda}(B)$ of non-absolute type.

For arbitrary sequence spaces $X$ and $Y$, the set $M(X, Y)$ defined by

$$
M(X, Y)=\left\{a=\left(a_{k}\right) \in \omega: a x=\left(a_{k} x_{k}\right) \in Y \forall x=\left(x_{k}\right) \in X\right\}
$$

is called the multiplier space of $X$ and $Y$. One can easily observe for a sequence space $Z$ with $Y \subset Z$ and $Z \subset X$ that the inclusions $M(X, Y) \subset M(X, Z)$ and $M(X, Y) \subset M(Z, Y)$ hold, respectively.

With the notation of (5.1), the $\alpha-, \beta$-, and $\gamma$-duals of a sequence space $X$, which are respectively, denoted by $X^{\alpha}, X^{\beta}$, and $X^{\gamma}$, are defined by

$$
X^{\alpha}=M\left(X, \ell_{1}\right), \quad X^{\beta}=M(X, c s), \quad X^{\gamma}=M(X, b s) .
$$

It is clear that $X^{\alpha} \subset X^{\beta} \subset X^{\gamma}$. Also it can be obviously seen that the inclusions $X^{\alpha} \subset Y^{\alpha}$, $X^{\beta} \subset Y^{\beta}$, and $X^{\gamma} \subset Y^{\gamma}$ hold whenever $Y \subset X$. 
Now, we may begin with quoting the following lemmas (see [25]) which are needed to prove Theorems 5.5 to 5.8 .

Lemma 5.1. $A=\left(a_{n k}\right) \in\left(c_{0}: \ell_{1}\right)=\left(c: \ell_{1}\right)$ if and only if

$$
\sup _{K \in \mathscr{F}} \sum_{n}\left|\sum_{k \in K} a_{n k}\right|<\infty \text {. }
$$

Lemma 5.2. $A=\left(a_{n k}\right) \in\left(c_{0}: c\right)$ if and only if

$$
\begin{gathered}
\lim _{n \rightarrow \infty} a_{n k}=\alpha_{k} \quad \text { for each fixed } k \in \mathbb{N}, \\
\sup _{n \in \mathbb{N}} \sum_{k}\left|a_{n k}\right|<\infty .
\end{gathered}
$$

Lemma 5.3. $A=\left(a_{n k}\right) \in(c: c)$ if and only if (5.4) and (5.5) hold, and

$$
\lim _{n \rightarrow \infty} \sum_{k} a_{n k} \text { exists. }
$$

Lemma 5.4. $A=\left(a_{n k}\right) \in\left(c: \ell_{\infty}\right)=\left(c_{0}: \ell_{\infty}\right)$ if and only if (5.5) holds.

Now, we prove the following result.

Theorem 5.5. The $\alpha$-dual of the spaces $c_{0}^{\lambda}(B)$ and $c^{\lambda}(B)$ is the set

$$
h_{1}^{\lambda}=\left\{a=\left(a_{n}\right) \in \omega: \sup _{K \in \mathcal{F}} \sum_{n}\left|\sum_{k \in K} h_{n k}^{\curlywedge}\right|<\infty\right\},
$$

where the matrix $H^{\lambda}=\left(h_{n k}^{\lambda}\right)$ is defined via the sequence $a=\left(a_{n}\right) \in \omega$ by

$$
h_{n k}^{(\lambda)}= \begin{cases}\left(\frac{-s}{r}\right)^{n-k}\left[\frac{\lambda_{k}}{r\left(\lambda_{k}-\lambda_{k-1}\right)}+\frac{\lambda_{k}}{s\left(\lambda_{k+1}-\lambda_{k}\right)}\right] a_{n}, & k<n, \\ \frac{\lambda_{n}}{r\left(\lambda_{n}-\lambda_{n-1}\right)} a_{n,} & k=n, \\ 0, & k>n\end{cases}
$$

for all $n, k \in \mathbb{N}$.

Proof. Let $a=\left(a_{n}\right) \in \omega$. Then, by bearing in mind the relations (2.11) and (2.14), it is immediate that the equality

$$
a_{n} x_{n}=\sum_{k=0}^{n} \frac{1}{r}\left(\frac{-s}{r}\right)^{n-k} \sum_{i=k-1}^{k}(-1)^{k-i} \frac{\lambda_{i}}{\lambda_{k}-\lambda_{k-1}} a_{n} y_{i}=H_{n}^{\lambda}(y)
$$


holds for all $n \in \mathbb{N}$. Thus, we observe by (5.9) that $a x=\left(a_{n} x_{n}\right) \in \ell_{1}$ whenever $x=\left(x_{k}\right) \in c_{0}^{\lambda}(B)$ or $c^{\lambda}(B)$ if and only if $H^{\lambda} y \in \ell_{1}$ whenever $y=\left(y_{k}\right) \in c_{0}$ or $c$. This means that the sequence $a=\left(a_{n}\right) \in\left\{c_{0}^{\lambda}(B)\right\}^{\alpha}$ or $a=\left(a_{n}\right) \in\left\{c^{\lambda}(B)\right\}^{\alpha}$ if and only if $H^{\lambda} \in\left(c_{0}: \ell_{1}\right)=\left(c: \ell_{1}\right)$. We therefore obtain by Lemma 5.4 with $H^{\lambda}$ instead of $A$ that $a=\left(a_{n}\right) \in\left\{c_{0}^{\lambda}(B)\right\}^{\alpha}=\left\{c^{\lambda}(B)\right\}^{\alpha}$ if and only if

$$
\sup _{K \in \mathcal{F}} \sum_{n}\left|\sum_{k \in K} h_{n k}^{\lambda}\right|<\infty
$$

which leads us to the consequence that $\left\{c_{0}^{\lambda}(B)\right\}^{\alpha}=\left\{c^{\lambda}(B)\right\}^{\alpha}=h_{1}^{\lambda}$. This completes the proof.

Theorem 5.6. Define the sets $h_{2}^{\lambda}, h_{3}^{\lambda}, h_{4}^{\lambda}$, and $h_{5}^{\lambda}$, as follows:

$$
\begin{aligned}
h_{2}^{\lambda} & =\left\{a=\left(a_{k}\right) \in \omega: \sum_{j=k}^{\infty}\left(\frac{-s}{r}\right)^{n-j} a_{j} \text { exists for each } \mathrm{k} \in \mathbb{N}\right\}, \\
h_{3}^{\lambda} & =\left\{a=\left(a_{k}\right) \in \omega: \sup _{n \in \mathbb{N}} \sum_{k=0}^{n-1}\left|\widehat{a}_{k}(n)\right|<\infty\right\}, \\
h_{4}^{\lambda} & =\left\{a=\left(a_{k}\right) \in \omega: \sup _{n \in \mathbb{N}}\left|\frac{1}{r} \frac{\lambda_{k}}{\left(\lambda_{k}-\lambda_{k-1}\right)} a_{k}\right|<\infty\right\}, \\
h_{5}^{\lambda} & =\left\{a=\left(a_{k}\right) \in \omega: \sum_{k} \frac{1}{r} \sum_{j=0}^{k}\left(\frac{-s}{r}\right)^{j} a_{k} \text { converges }\right\}, \quad \text { where } \\
\widehat{a}_{k}(n) & =\lambda_{k}\left[\frac{a_{k}}{r\left(\lambda_{k}-\lambda_{k-1}\right)}+\left(\frac{1}{r\left(\lambda_{k}-\lambda_{k-1}\right)}+\frac{1}{s\left(\lambda_{k+1}-\lambda_{k}\right)}\right) \sum_{j=k+1}^{n}\left(\frac{-s}{r}\right)^{n-j} a_{j}\right] \quad \text { for } \mathrm{k}<\mathrm{n} .
\end{aligned}
$$

Then $\left\{c_{0}^{\lambda}(B)\right\}^{\beta}=h_{2}^{\lambda} \cap h_{3}^{\lambda} \cap h_{4}^{\lambda}$ and $\left\{c^{\lambda}(B)\right\}^{\beta}=h_{2}^{\lambda} \cap h_{3}^{\lambda} \cap h_{4}^{\lambda} \cap h_{5}^{\lambda}$. 
Proof. Consider the equality

$$
\begin{aligned}
\sum_{k=0}^{n} a_{k} x_{k}= & \sum_{k=0}^{n}\left\{\sum_{j=0}^{k} \frac{1}{r}\left(\frac{-s}{r}\right)^{k-j}\left[\sum_{i=j-1}^{j}(-1)^{j-i} \frac{\lambda_{i}}{\lambda_{j}-\lambda_{j-1}} y_{i}\right]\right\} a_{k} \\
= & \sum_{k=0}^{n-1} \lambda_{k}\left[\frac{a_{k}}{r\left(\lambda_{k}-\lambda_{k-1}\right)}+\left(\frac{1}{r\left(\lambda_{k}-\lambda_{k-1}\right)}+\frac{1}{s\left(\lambda_{k+1}-\lambda_{k}\right)}\right) \sum_{j=k+1}^{n}\left(\frac{-s}{r}\right)^{n-j} a_{j}\right] y_{k} \\
& +\frac{1}{r} \frac{\lambda_{n}}{\left(\lambda_{n}-\lambda_{n-1}\right)} a_{n} y_{n} \\
= & \sum_{k=0}^{n-1} \widehat{a}_{k}(n) y_{k}+\frac{1}{r} \frac{\lambda_{n}}{\left(\lambda_{n}-\lambda_{n-1}\right)} a_{n} y_{n} \\
= & T_{n}^{\lambda}(y) \quad \forall n \in \mathbb{N},
\end{aligned}
$$

where the matrix $T^{\lambda}=\left(t_{n k}^{\lambda}\right)$ is defined by

$$
t_{n k}^{\lambda}:= \begin{cases}\widehat{a}_{k}(n), & k<n, \\ \frac{1}{r} \frac{\lambda_{n}}{\left(\lambda_{n}-\lambda_{n-1}\right)} a_{n}, & k=n, \\ 0, & k>n\end{cases}
$$

for all $k, n \in \mathbb{N}$. Then, we deduce by (5.12) that $a x=\left(a_{k} x_{k}\right) \in c s$ whenever $x=\left(x_{k}\right) \in c_{0}^{\lambda}(B)$ if and only if $T^{\lambda} y \in c$ whenever $y=\left(y_{k}\right) \in c_{0}$. This means that $a=\left(a_{k}\right) \in\left\{c_{0}^{\lambda}(B)\right\}^{\beta}$ if and only if $T^{\lambda} \in\left(c_{0}: c\right)$. Therefore, by using Lemma 5.2, we derive from (5.4) and (5.5) that

$$
\begin{gathered}
\sum_{j=k+1}^{\infty}\left(\frac{-s}{r}\right)^{n-j} a_{j} \text { exists for each } k \in \mathbb{N}, \\
\sup _{n \in \mathbb{N}} \sum_{k=0}^{n-1}\left|\widehat{a}_{k}(n)\right|<\infty, \\
\sup _{n \in \mathbb{N}}\left|\frac{1}{r} \frac{\lambda_{n}}{\left(\lambda_{n}-\lambda_{n-1}\right)} a_{n}\right|<\infty .
\end{gathered}
$$

Therefore, we conclude that $\left\{c_{0}^{\lambda}(B)\right\}^{\beta}=h_{2}^{\lambda} \cap h_{3}^{\lambda} \cap h_{4}^{\lambda}$.

Similarly, we deduce from Lemma 5.3 with (5.12) that $a=\left(a_{k}\right) \in\left\{c^{\mathcal{\lambda}}(B)\right\}^{\beta}$ if and only if $T^{\lambda} \in(c: c)$. Therefore, we derive from (5.4) and (5.5) that (5.14), (5.15) hold.

Further, with a simple calculation one can easily see that the equality

$$
\sum_{k=0}^{n} \frac{1}{r} \sum_{j=0}^{k}\left(\frac{-s}{r}\right)^{j} a_{k}=\sum_{k=0}^{n-1} \widehat{a}_{k}(n)+\frac{1}{r} \frac{\lambda_{n}}{\left(\lambda_{n}-\lambda_{n-1}\right)} a_{n}=\sum_{k} t_{n k}^{\lambda}
$$


holds for all $n \in \mathbb{N}$. Consequently, from (5.6) we obtain that

$$
\left\{\frac{1}{r} \sum_{j=0}^{k}\left(\frac{-s}{r}\right)^{j} a_{k}\right\} \in c s .
$$

Hence, we deduce that $\left\{c^{\lambda}(B)\right\}^{\beta}=h_{2}^{\lambda} \cap h_{3}^{\lambda} \cap h_{4}^{\lambda} \cap h_{5}^{\lambda}$. This completes the proof.

Remark 5.7. We may note by combining (5.17) with the conditions (5.15) that $\left\{\sum_{j=0}^{k}(-s / r)^{j} a_{k} / r\right\} \in b s$ for every sequence $a=\left(a_{k}\right) \in\left\{c_{0}^{\lambda}(B)\right\}^{\beta}$.

Finally, we close this section with the following theorem which determines the $\gamma$-dual of the spaces $c_{0}^{\lambda}(B)$ and $c^{\lambda}(B)$ :

Theorem 5.8. The $\gamma$-duals of the spaces $c_{0}^{\lambda}(B)$ and $c^{\lambda}(B)$ are the set $h_{3}^{\lambda} \cap h_{4}^{\lambda}$.

Proof. The proof of this result follows the same lines that in the proof of Theorem 5.6 using Lemma 5.4 instead of Lemma 5.2.

\section{Certain Matrix Mappings Related to the Spaces $c_{0}^{\lambda}(B)$ and $c^{\lambda}(B)$}

In this final section, we characterize the matrix classes $\left(c^{\lambda}(B): \ell_{p}\right),\left(c_{0}^{\lambda}(B): \ell_{p}\right),\left(c^{\lambda}(B): c\right)$, $\left(c^{\lambda}(B): c_{0}\right),\left(c_{0}^{\lambda}(B): c\right)$, and $\left(c_{0}^{\mathcal{\lambda}}(B): c_{0}\right)$, where $1 \leq p \leq \infty$. Also, by means of a given basic lemma, we derive the characterizations of some other classes involving the Euler, difference, Riesz, and Cesàro sequence spaces.

For an infinite matrix $A=\left(a_{n k}\right)$, we write for brevity that

$$
\begin{aligned}
\widehat{a}_{n k}(m) & =\lambda_{k}\left[\frac{a_{n k}}{r\left(\lambda_{k}-\lambda_{k-1}\right)}+\left(\frac{1}{r\left(\lambda_{k}-\lambda_{k-1}\right)}+\frac{1}{s\left(\lambda_{k+1}-\lambda_{k}\right)}\right) \sum_{j=k+1}^{m}\left(\frac{-s}{r}\right)^{n-j} a_{n j}\right] \text { if } k<m, \\
\widehat{a}_{n k} & =\lambda_{k}\left[\frac{a_{n k}}{r\left(\lambda_{k}-\lambda_{k-1}\right)}+\left(\frac{1}{r\left(\lambda_{k}-\lambda_{k-1}\right)}+\frac{1}{s\left(\lambda_{k+1}-\lambda_{k}\right)}\right) \sum_{j=k+1}^{\infty}\left(\frac{-s}{r}\right)^{n-j} a_{n j}\right]
\end{aligned}
$$

for all $k, m, n \in \mathbb{N}$ provided the convergence of the series.

The following lemmas will be needed in proving our main results.

Lemma 6.1 (see [24, page 57]). The matrix mappings between the BK-spaces are continuous.

Lemma 6.2 (see [25, pages 7-8]). $A=\left(a_{n k}\right) \in\left(c: \ell_{p}\right)$ if and only if

$$
\sup _{K \in \mathcal{F}} \sum_{n}\left|\sum_{k \in K} a_{n k}\right|^{p}<\infty, \quad(1 \leq p<\infty) .
$$


Lemma 6.3 (see [25, page 5]). $A=\left(a_{n k}\right) \in\left(c: c_{0}\right)$ if and only if (5.5) holds and

$$
\begin{gathered}
\lim _{n \rightarrow \infty} a_{n k}=0 \quad \forall k \in \mathbb{N}, \\
\lim _{n \rightarrow \infty} \sum_{k} a_{n k}=0 .
\end{gathered}
$$

Lemma 6.4 (see [25, page 5]). $A=\left(a_{n k}\right) \in\left(c_{0}: c_{0}\right)$ if and only if (5.5) and (6.3) hold.

Now, we give the following results on the matrix transformations.

Theorem 6.5. Let $A=\left(a_{n k}\right)$ be an infinite matrix over the complex field. Then, the following statements hold.

(i) Let $(1 \leq p<\infty)$. Then, $A \in\left(c^{\lambda}(B): \ell_{p}\right)$ if and only if

$$
\begin{gathered}
\sum_{j=k+1}^{\infty}\left(\frac{-s}{r}\right)^{n-j} a_{n j} \text { exists for each fixed } \mathrm{k} \in \mathbb{N}, \\
\sup _{K \in \mathcal{F}} \sum_{n}\left|\sum_{k \in K} \widehat{a}_{n k}\right|^{p}<\infty, \\
\sup _{m \in \mathbb{N}} \sum_{k=0}^{m-1}\left|\widehat{a}_{n k}(m)\right|<\infty, \quad \forall n \in \mathbb{N}, \\
\left\{\frac{1}{r} \sum_{j=0}^{k}\left(\frac{-s}{r}\right)^{j} a_{n k}\right\}_{k=0}^{\infty} \in c s \quad \text { for each fixed } \mathrm{n} \in \mathbb{N}, \\
\lim _{k \rightarrow \infty} \frac{\lambda_{k}}{r\left(\lambda_{k}-\lambda_{k-1}\right)} a_{n k}=a_{n} \quad \text { for each fixed } \mathrm{n} \in \mathbb{N}, \\
\left(a_{n}\right) \in \ell_{p} .
\end{gathered}
$$

(ii) $A \in\left(c^{\lambda}(B): \ell_{\infty}\right)$ if and only if (6.7) and (6.8) hold, and

$$
\begin{gathered}
\sup _{n \in \mathbb{N}} \sum_{k}\left|\widehat{a}_{n k}\right|<\infty, \\
\left(a_{n}\right) \in \ell_{\infty} .
\end{gathered}
$$

Proof. Suppose that the conditions (6.4)-(6.9) hold and take any $x=\left(x_{k}\right) \in c^{\lambda}(B)$. Then, we have by Theorem 5.6 that $\left\{a_{n k}\right\}_{k \in \mathbb{N}} \in\left\{c^{\lambda}(B)\right\}^{\beta}$ for all $n \in \mathbb{N}$ and this implies that the $A$ transform of $x$ exists. Also, it is clear that the associated sequence $y=\left(y_{k}\right)$ is in the space $c$ and hence $y_{k} \rightarrow l$ as $k \rightarrow \infty$ for some suitable $l$. Further, it follows by combining Lemma 6.2 with (6.5) that the matrix $\widehat{A}=\left(\widehat{a}_{n k}\right)$ is in the class $\left(c: \ell_{p}\right)$, where $1 \leq p<\infty$. 
Let us now consider the following equality derived by using the relation (2.11) from the $m$ th partial sum of the series $\sum_{k} a_{n k} x_{k}$ :

$$
\sum_{k=0}^{m} a_{n k} x_{k}=\sum_{k=0}^{m-1} \widehat{a}_{n k}(m) y_{k}+\frac{\lambda_{m}}{r\left(\lambda_{m}-\lambda_{m-1}\right)} a_{n m} y_{m}, \quad \forall n, m \in \mathbb{N}
$$

Then, since $y \in c$ and $\widehat{A} \in\left(c: \ell_{p}\right)$, the series $\sum_{k} \widehat{a}_{n k} y_{k}$ converges for every $n \in \mathbb{N}$. Furthermore, it follows by (6.4) that the series $\sum_{j=k}^{\infty}(-s / r)^{n-j} a_{n j}$ converges for all $n, k \in \mathbb{N}$ and hence $\widehat{a}_{n k}(m) \rightarrow \widehat{a}_{n k}$ as $m \rightarrow \infty$. Therefore, if we pass to limit in (6.12) as $m \rightarrow \infty$ then we obtain by (6.8) that

$$
\sum_{k} a_{n k} x_{k}=\sum_{k} \widehat{a}_{n k} y_{k}+l a_{n,} \quad \forall n \in \mathbb{N}
$$

which can be written as follows:

$$
A_{n}(x)=\widehat{A}_{n}(y)+l a_{n}, \quad \forall n \in \mathbb{N}
$$

This yields by taking $p$-norm that

$$
\|A x\|_{p} \leq\|\widehat{A} y\|_{p}+|l|\left\|\left(a_{n}\right)\right\|_{p}<\infty
$$

which leads us to the consequence that $A x \in \ell_{p}$. Hence, $A \in\left(c^{\lambda}(B): \ell_{p}\right)$.

Conversely, suppose that $A \in\left(c^{\lambda}(B): \ell_{p}\right)$, where $1 \leq p<\infty$. Then $\left\{a_{n k}\right\}_{k \in \mathbb{N}} \in\left\{c^{\lambda}(B)\right\}^{\beta}$ for all $n \in \mathbb{N}$ which implies with Theorem 5.6 that the conditions (6.6) and (6.7) are necessary.

On the other hand, since $c^{\lambda}(B)$ and $\ell_{p}$ are $B K$-spaces, we have by Lemma 6.1 that there is a constant $M>0$ such that

$$
\|A x\|_{p} \leq M\|x\|_{c^{\lambda}(B)}
$$

holds for all $x \in c^{\lambda}(B)$. Now, $K \in \mathcal{F}$. Then, the sequence $z=\sum_{k \in K} b^{(k)}(\lambda)$ is in $c^{\lambda}(B)$, where the sequence $b^{(k)}(\lambda)=\left\{b_{n}^{(k)}(\lambda)\right\}_{n \in \mathbb{N}}$ is defined by (4.2) for every fixed $k \in \mathbb{N}$.

Since $\widehat{\Lambda}\left(b^{(k)}(\lambda)\right)=e^{(k)}$ for each fixed $k \in \mathbb{N}$, we have

$$
\|z\|_{\mathcal{C}^{\lambda}(B)}=\|\widehat{\Lambda}(z)\|_{\infty}=\left\|\sum_{k \in K} \widehat{\Lambda}\left(b^{(k)}(\lambda)\right)\right\|_{\infty}=\left\|\sum_{k \in K} e^{(k)}\right\|_{\infty}=1 .
$$

Furthermore, for every $n \in \mathbb{N}$, we obtain by (4.2) that

$$
A_{n}(z)=\sum_{k \in K} A_{n}\left(b^{(k)}(\lambda)\right)=\sum_{k \in K} \sum_{j} a_{n j} b_{j}^{(k)}(\lambda)=\sum_{k \in K} \widehat{a}_{n k}
$$


Hence, since the inequality (6.16) is satisfied for the sequence $z \in c^{\lambda}(B)$, we have for any $K \in \mathcal{F}$ that

$$
\left(\sum_{n}\left|\sum_{k \in K} \widehat{a}_{n k}\right|^{p}\right)^{1 / p} \leq M
$$

which shows the necessity of (6.5). Thus, it follows by Lemma 6.2 that $\widehat{A}=\left(\widehat{a}_{n k}\right) \in\left(c: \ell_{p}\right)$.

Now, let $y=\left(y_{k}\right) \in c \backslash c_{0}$ and consider the sequence $x=\left(x_{k}\right)$ defined by (2.14) for every $k \in \mathbb{N}$. Then, $x \in c^{\lambda}(B)$ such that $y=\widehat{\Lambda}(x)$, that is, the sequences $x$ and $y$ are connected by the relation (2.11). Therefore, $A x$ and $\widehat{A} y$ exist. This leads us to the convergence of the series $\sum_{k} a_{n k} x_{k}$ and $\sum_{k} \widehat{a}_{n k} y_{k}$ for every $n \in \mathbb{N}$. We thus deduce that

$$
\lim _{m \rightarrow \infty} \sum_{k=0}^{m-1} \widehat{a}_{n k}(m) y_{k}=\sum_{k} \widehat{a}_{n k} y_{k}, \quad \forall n \in \mathbb{N}
$$

Consequently, we obtain from (6.12) as $m \rightarrow \infty$ that

$$
\lim _{m \rightarrow \infty} \frac{\lambda_{m}}{r\left(\lambda_{m}-\lambda_{m-1}\right)} a_{n m} y_{m} \text { exists for each fixed } n \in \mathbb{N}
$$

and since $y=\left(y_{k}\right) \in c \backslash c_{0}$, we conclude that

$$
\lim _{m \rightarrow \infty} \frac{\lambda_{m}}{r\left(\lambda_{m}-\lambda_{m-1}\right)} a_{n m} \text { exists for each fixed } n \in \mathbb{N}
$$

which shows the necessity of (6.8). Then relation (6.14) holds.

Finally, since $A x \in \ell_{p}$ and $\widehat{A} y \in \ell_{p}$, the necessity of (6.9) is immediate by (6.14). This completes the proof of Part (i) of the theorem.

Since Part (ii) can be proved by using the similar way that used in the proof of Part (i) with Lemma 5.4 instead of Lemma 6.2, we leave the details to the reader.

Remark 6.6. It is clear by (6.10) that the limit

$$
\lim _{m \rightarrow \infty} \sum_{k=0}^{m-1}\left|\widehat{a}_{n k}(m)\right|=\sum_{k}\left|\widehat{a}_{n k}\right|
$$

exists for each $n \in \mathbb{N}$. This just tells us that condition (6.10) implies condition (6.6).

Now, we may note that $\left(c_{0}: \ell_{p}\right)=\left(c: \ell_{p}\right)$ for $1 \leq p \leq \infty$, (see [25, pages 7-8]). Thus, by means of Theorem 5.6 and Lemmas 6.2 and 5.4, we immediately conclude the following theorem.

Theorem 6.7. Let $A=\left(a_{n k}\right)$ be an infinite matrix over the complex field. Then, the following statements hold. 
(i) Let $1 \leq p<\infty$. Then, $A \in\left(c_{0}^{\lambda}(B): \ell_{p}\right)$ if and only if (6.5) and (6.6) hold, and

$$
\begin{aligned}
& \sum_{j=k}^{\infty}\left(\frac{-s}{r}\right)^{n-j} a_{n j} \quad \text { exists for all } n, k \in \mathbb{N}, \\
& \left\{\frac{\lambda_{k}}{r\left(\lambda_{k}-\lambda_{k-1}\right)} a_{n k}\right\}_{k=0}^{\infty} \in \ell_{\infty}, \quad \forall n \in \mathbb{N} .
\end{aligned}
$$

(ii) $A \in\left(c_{0}^{\lambda}(B): \ell_{\infty}\right)$ if and only if (6.10) and (6.24) hold.

Proof. It is natural that the present theorem can be proved by the same technique used in the proof of Theorem 6.5, above, and so we omit the proof.

Theorem 6.8. $A=\left(a_{n k}\right) \in\left(c^{\lambda}(B): c\right)$ if and only if (6.7), (6.8), and (6.10) hold, and

$$
\begin{gathered}
\lim _{n \rightarrow \infty} a_{n}=a, \\
\lim _{n \rightarrow \infty} \widehat{a}_{n k}=\alpha_{k}, \quad \text { for each } \mathrm{k} \in \mathbb{N}, \\
\lim _{n \rightarrow \infty} \sum_{k} \widehat{a}_{n k}=\alpha .
\end{gathered}
$$

Proof. Suppose that $A$ satisfies the conditions (6.7), (6.8), (6.10), (6.25), (6.26), and (6.27) and take any $x \in c^{\lambda}(B)$. Then, since (6.10) implies (6.6), we have by Theorem 5.6 that $\left\{a_{n k}\right\}_{k \in \mathbb{N}} \in$ $\left\{C^{\curlywedge}(B)\right\}^{\beta}$ for all $n \in \mathbb{N}$ and hence $A x$ exists. We also observe from (6.10) and (6.26) that

$$
\sum_{j=0}^{k}\left|\alpha_{j}\right| \leq \sup _{m \in \mathbb{N}} \sum_{j}\left|\widehat{a}_{n k}\right|<\infty
$$

holds for every $k \in \mathbb{N}$. This implies that $\left(\alpha_{k}\right) \in \ell_{1}$ and hence the series $\sum_{k} \alpha_{k}\left(y_{k}-l\right)$ converges, where $y=\left(y_{k}\right) \in c$ is the sequence connected with $x=\left(x_{k}\right)$ by the relation (2.11) such that $y_{k} \rightarrow l$ as $k \rightarrow \infty$. Further it is obvious by combining Lemma 5.3 with the condition (6.10), (6.26), and (6.27) that the matrix $\widehat{A}=\left(\widehat{a}_{n k}\right)$ is in the class $(c: c)$.

Now reasoning as in the proof of Theorem 6.5, we obtain that the relation (6.13) holds which can be written as follows:

$$
\sum_{k} a_{n k} x_{k}=\sum_{k} \widehat{a}_{n k}\left(y_{k}-l\right)+l \sum_{k} \widehat{a}_{n k}+l a_{n}, \quad \forall n \in \mathbb{N} .
$$

In this situation, we see by passing to the limit in (6.29) as $n \rightarrow \infty$ that the first term on the right tends to $\sum_{k} \alpha_{k}\left(y_{k}-l\right)$ by (6.10) and (6.26). The second term tends to $l \alpha$ by (6.27) and the last term to la by (6.25). Consequently, we have

$$
\lim _{n \rightarrow \infty} A_{n}(x)=\sum_{k} \alpha_{k}\left(y_{k}-l\right)+l(\alpha+a)
$$

which shows that $A x \in c$, that is to say that $A \in\left(c^{\lambda}(B): c\right)$. 
Conversely, Suppose that $A \in\left(c^{\lambda}(B): c\right)$. Then, since the inclusion $c \subset \ell_{\infty}$ holds, it is trivial that $A \in\left(c^{\lambda}(B): \ell_{\infty}\right)$. Therefore, the necessity of the conditions (6.7), (6.8), and (6.10) are obvious from Theorem 6.5. Further, consider the sequence $b^{(k)}(\lambda)=\left\{b_{n}^{(k)}(\lambda)\right\}_{n \in \mathbb{N}} \in c^{\lambda}(B)$ defined by (4.2) for every fixed $k \in \mathbb{N}$. Then, it is easily seen that $A b^{(k)}(\lambda)=\left\{\widehat{a}_{n k}\right\}_{n \in \mathbb{N}}$ and hence $\left\{\widehat{a}_{n k}\right\}_{n \in \mathbb{N}} \in c$ for every $k \in \mathbb{N}$ which shows the necessity of (6.26). Let $z=\sum_{k} b^{(k)}(\lambda)$. Then, since the linear transformation $T: c^{\lambda}(B) \rightarrow c$, defined as in the proof of Theorem 2.3 by analogy, is continuous and $\widehat{\Lambda}\left(b^{(k)}(\lambda)\right)=e^{(k)}$ for each fixed $k \in \mathbb{N}$, we obtain that

$$
\widehat{\Lambda}_{n}(z)=\sum_{k} \widehat{\Lambda}_{n}\left(b^{(k)}(\lambda)\right)=\sum_{k} \delta_{n k}=1 \quad \text { for each } n \in \mathbb{N}
$$

which shows that $\widehat{\Lambda}(z)=e \in c$ and hence $z \in c^{\lambda}(B)$. On the other hand, since $c^{\mathcal{\lambda}}(B)$ and $c$ are the $B K$-spaces, Lemma 6.1 implies the continuity of the matrix mapping $A: c^{\mathcal{l}}(B) \rightarrow c$. Thus, we have for every $n \in \mathbb{N}$ that

$$
A_{n}(z)=\sum_{k} A_{n}\left(b^{(k)}(\lambda)\right)=\sum_{k} \widehat{a}_{n k}
$$

This shows the necessity of (6.27).

Now, it follows by (6.10), (6.26), and (6.27) with Lemma 5.3 that $\widehat{A}=\left(\widehat{a}_{n k}\right) \in(c: c)$. So by (6.7), and (6.8), relation (6.14) holds for all $x \in c^{\lambda}(B)$ and $y \in c$, and $x$ and $y$ are connected by relation (2.11), where $y_{k} \rightarrow l(k \rightarrow \infty)$.

Lastly, since $A x \in \mathcal{C}$ and $\widehat{A} x \in c$; the necessity of (6.25) is immediate by (6.14). This step concludes the proof.

Theorem 6.9. $A=\left(a_{n k}\right) \in\left(c^{\lambda}(B): c_{0}\right)$ if and only if (6.7), (6.8), and (6.10) hold, and

$$
\begin{gathered}
\lim _{n \rightarrow \infty} a_{n}=0, \\
\lim _{n \rightarrow \infty} \widehat{a}_{n k}=0 \quad \text { for each } \mathrm{k} \in \mathbb{N}, \\
\lim _{n \rightarrow \infty} \sum_{k} \widehat{a}_{n k}=0 .
\end{gathered}
$$

Proof. Since the present theorem can be proved by the similar way used in the proof of Theorem 6.8 with Lemma 6.3 instead of Lemma 5.3, we omit the detailed proof.

Theorem 6.10. $A=\left(a_{n k}\right) \in\left(c_{0}^{\lambda}(B): c\right)$ if and only if (6.10), (6.24), and (6.26) hold.

Proof. This is similarly obtained by using Lemma 5.2, Theorem 5.6, and Part (ii) of Theorem 6.7.

Theorem 6.11. $A=\left(a_{n k}\right) \in\left(c_{0}^{\lambda}(B): c_{0}\right)$ if and only if (6.10) and (6.24) hold, and (6.26) also holds with $\alpha_{k}=0$ for all $k \in \mathbb{N}$.

Proof. This is immediate by Lemma 6.4, Theorems 5.6 and 6.10 . 


\section{Conclusion}

Let $v$ denotes any of the classical sequence spaces $\ell_{\infty}, c$, or $c_{0}$. Then, $v(\Delta)$ consisting of the sequences $x=\left(x_{k}\right)$ such that $\Delta x=\left(x_{k}-x_{k+1}\right) \in v$ is called as the difference sequence space which was introduced by Kizmaz [19]. Kizmaz [19] proved that $v(\Delta)$ is a Banach space with the norm $\|x\|_{\Delta}=\left|x_{1}\right|+\|\Delta x\|_{\infty}$, where $x=\left(x_{k}\right) \in v(\Delta)$ and the inclusion relation $v \subset v(\Delta)$ strictly holds. He also determined the $\alpha-, \beta-$, and $\gamma$-duals of the difference spaces and characterized the classes $(v(\Delta): \mu)$ and $(\mu: v(\Delta))$ of infinite matrices, where $\nu, \mu \in\left\{\ell_{\infty}, c\right\}$. Following Kızmaz [19], Sarıgöl [26] extended the difference space $v(\Delta)$ to the space $v\left(\Delta_{r}\right)$ defined by

$$
\mathcal{v}\left(\Delta_{r}\right):=\left\{x=\left(x_{k}\right) \in \omega: \Delta_{r} x=\left\{k^{r}\left(x_{k}-x_{k+1}\right)\right\} \in \mathcal{v} \text { for } r<1\right\}
$$

and computed the $\alpha-, \beta$-, and $\gamma$-duals of the space $v\left(\Delta_{r}\right)$, where $v \in\left\{\ell_{\infty}, c, c_{0}\right\}$. It is easily seen that $v\left(\Delta_{r}\right) \subset v(\Delta)$, if $0<r<1$ and $v(\Delta) \subset v\left(\Delta_{r}\right)$, if $r<0$. Recently, the difference spaces $b v_{p}$ consisting of the sequences $x=\left(x_{k}\right)$ such that $\left(x_{k}-x_{k-1}\right) \in \ell_{p}$ have been studied in the case $0<p<1$ by Altay and Başar [27] and in the case $1 \leq p \leq \infty$ by Başar and Altay [18], Çolak et al. [28], and Malkowsky et al. [29]. Quite recently, Mursaleen and Noman have introduced the spaces $c^{\lambda}$ and $c_{0}^{\lambda}$ of $\lambda$-convergent and $\lambda$-null sequences and nextly studied the difference spaces $c^{\lambda}(\Delta)$ and $c_{0}^{\lambda}(\Delta)$ in [21, 22], respectively. Of course, there is a wide literature concerning the difference sequence spaces. By the domain of the generalized difference matrix $B(r, s)$ in the spaces of $\lambda$-convergent and $\lambda$-null sequences we have generalized the difference spaces $c^{\lambda}(\Delta)$ and $c_{0}^{\lambda}(\Delta)$ defined by Mursaleen and Noman [21]. Since the generalized difference matrix $B(r, s)$ reduces, in the special case $r=1, s=-1$, to the usual difference matrix $\Delta$; our results are more general and more comprehensive than the corresponding results of Mursaleen and Noman [21].

Since the difference spaces of $\lambda$-bounded and absolutely $\lambda p$-summable sequences are not studied, the domain of both the difference matrix $\Delta$, and the generalized difference matrix $B(r, s)$ in those spaces are still open. So, it is meaningful to fill this gap.

\section{Acknowledgments}

The authors have benefited a lot from discussion on the main results of the earlier version of the paper with Professor Bilâl Altay, Department of Mathematical Education, Faculty of Education, İnönü University, Turkey. So, they wish to express their gratitude for his many helpful suggestions and interesting comments.

\section{References}

[1] B. Choudhary and S. Nanda, Functional Analysis with Applications, John Wiley \& Sons, New Delhi, India, 1989.

[2] B. Altay and F. Başar, "On the paranormed Riesz sequence spaces of non-absolute type," Southeast Asian Bulletin of Mathematics, vol. 26, no. 5, pp. 701-715, 2003.

[3] B. Altay and F. Başar, "Some Euler sequence spaces of nonabsolute type," Ukrainian Mathematical Journal, vol. 57, no. 1, pp. 3-17, 2005.

[4] B. Altay, F. Başar, and M. Mursaleen, “On the Euler sequence spaces which include the spaces $l_{p}$ and $l_{\infty}$. I," Information Sciences, vol. 176, no. 10, pp. 1450-1462, 2006.

[5] C. Aydın and F. Başar, "On the new sequence spaces which include the spaces $c_{0}$ and $c$," Hokkaido Mathematical Journal, vol. 33, no. 2, pp. 383-398, 2004. 
[6] C. Aydın and F. Başar, "Some new paranormed sequence spaces," Information Sciences, vol. 160, no. 1-4, pp. 27-40, 2004.

[7] C. Aydın and F. Başar, "Some new sequence spaces which include the spaces $l_{P}$ and $l_{\infty}$, " Demonstratio Mathematica, vol. 38, no. 3, pp. 641-656, 2005.

[8] E. Malkowsky, "Recent results in the theory of matrix transformations in sequence spaces," Matematichki Vesnik, vol. 49, no. 3-4, pp. 187-196, 1997.

[9] E. Malkowsky and E. Savas, "Matrix transformations between sequence spaces of generalized weighted means," Applied Mathematics and Computation, vol. 147, no. 2, pp. 333-345, 2004.

[10] P. N. Ng and P. Y. Lee, "Cesàro sequence spaces of non-absolute type," Commentationes Mathematicae. Prace Matematyczne, vol. 20, no. 2, pp. 429-433, 1978.

[11] M. Şengönül and F. Başar, "Some new Cesàro sequence spaces of non-absolute type which include the spaces $c_{0}$ and $c_{,}$" Soochow Journal of Mathematics, vol. 31, no. 1, pp. 107-119, 2005.

[12] A. Sönmez, "Some new sequence spaces derived by the domain of the triple band matrix," Computers $\mathcal{E}$ Mathematics with Applications, vol. 62, no. 2, pp. 641-650, 2011.

[13] A. Sönmez, "Almost convergence and triple band matrix," Mathematical and Computer Modelling. In press.

[14] C. S. Wang, “On Nörlund sequence spaces," Tamkang Journal of Mathematics, vol. 9, no. 2, pp. 269-274, 1978.

[15] M. Başarır, “On some new sequence spaces and related matrix transformations," Indian Journal of Pure and Applied Mathematics, vol. 26, no. 10, pp. 1003-1010, 1995.

[16] B. Altay, F. Başar, and E. Malkowsky, "Matrix transformations on some sequence spaces related to strong Cesàro summability and boundedness," Applied Mathematics and Computation, vol. 211, no. 2, pp. 255-264, 2009.

[17] C. Aydın and F. Başar, "Some new difference sequence spaces," Applied Mathematics and Computation, vol. 157, no. 3, pp. 677-693, 2004.

[18] F. Başar and B. Altay, "On the space of sequences of $p$-bounded variation and related matrix mappings," Ukrainian Mathematical Journal, vol. 55, no. 1, pp. 136-147, 2003.

[19] H. Kizmaz, "On certain sequence spaces," Canadian Mathematical Bulletin, vol. 24, no. 2, pp. 169-176, 1981.

[20] E. Malkowsky, M. Mursaleen, and S. Suantai, "The dual spaces of sets of difference sequences of order $m$ and matrix transformations," Acta Mathematica Sinica, vol. 23, no. 3, pp. 521-532, 2007.

[21] M. Mursaleen and A. K. Noman, "On some new difference sequence spaces of non-absolute type," Mathematical and Computer Modelling, vol. 52, no. 3-4, pp. 603-617, 2010.

[22] M. Mursaleen and A. K. Noman, "On the spaces of $\lambda$-convergent and bounded sequences," Thai Journal of Mathematics, vol. 8, no. 2, pp. 311-329, 2010.

[23] I. J. Maddox, Elements of Functional Analysis, Cambridge University Press, Cambridge, UK, 2nd edition, 1988.

[24] A. Wilansky, Summability Throught Functional Analysis, vol. 85 of North-Holland Mathematics Studies, North-Holland, Oxford, UK, 1984.

[25] M. Stieglitz and H. Tietz, "Matrixtransformationen von Folgenräumen. Eine Ergebnisübersicht," Mathematische Zeitschrift, vol. 154, no. 1, pp. 1-16, 1977.

[26] M. A. Sarı̈öl, “On difference sequence spaces," Journal of Karadeniz Technical University, vol. 10, pp. 63-71, 1987.

[27] B. Altay and F. Başar, "The fine spectrum and the matrix domain of the difference operator $\Delta$ on the sequence space $l_{p},(0<P<1)$," Communications in Mathematical Analysis, vol. 2, no. 2, pp. 1-11, 2007.

[28] R. Çolak, M. Et, and E. Malkowsky, Some Topics of Sequence Spaces, Lecture Notes in Mathematics, Firat University Press, 2004.

[29] E. Malkowsky, V. Rakočević, and S. Živković, "Matrix transformations between the sequence space $b v^{p}$ and certain BK spaces," Bulletin. Classe des Sciences Mathématiques et Naturelles. Sciences Mathématiques, no. 27, pp. 33-46, 2002. 


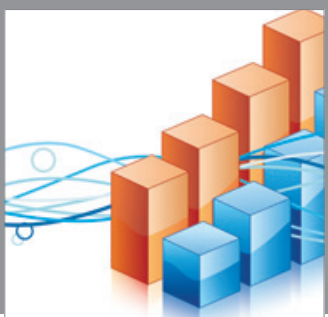

Advances in

Operations Research

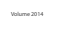

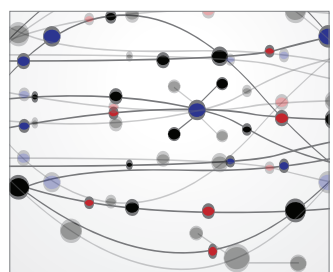

\section{The Scientific} World Journal
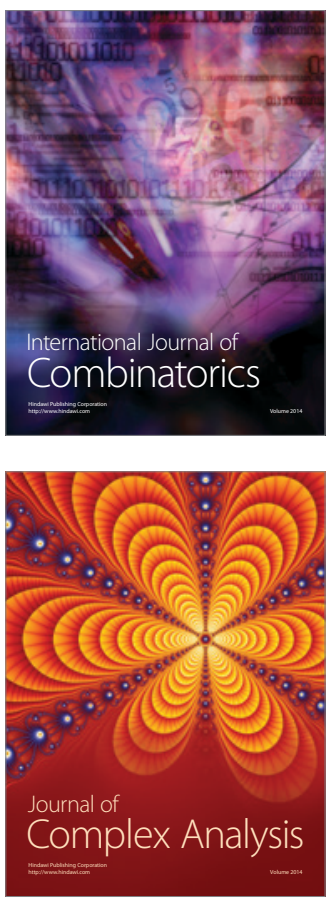

International Journal of

Mathematics and

Mathematical

Sciences
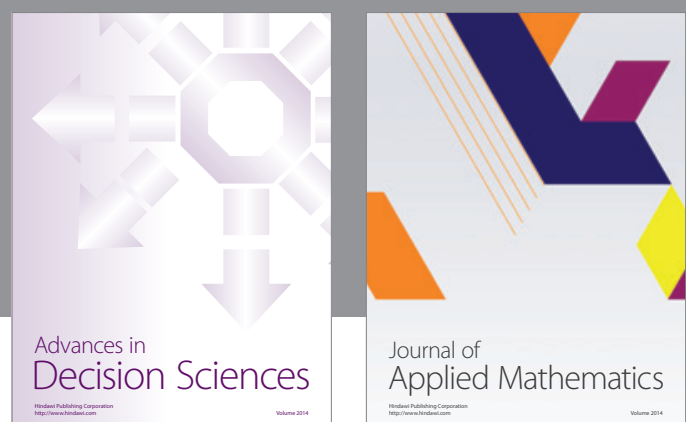

Journal of

Applied Mathematics
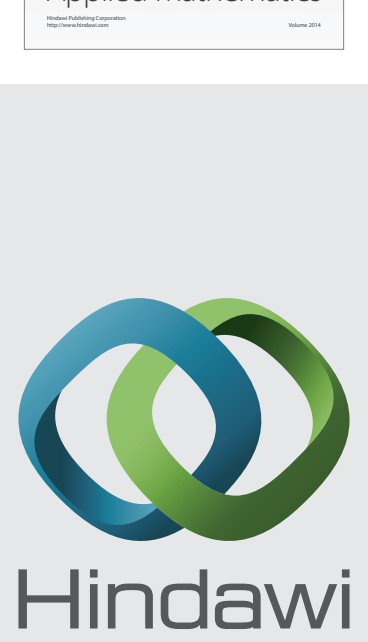

Submit your manuscripts at http://www.hindawi.com
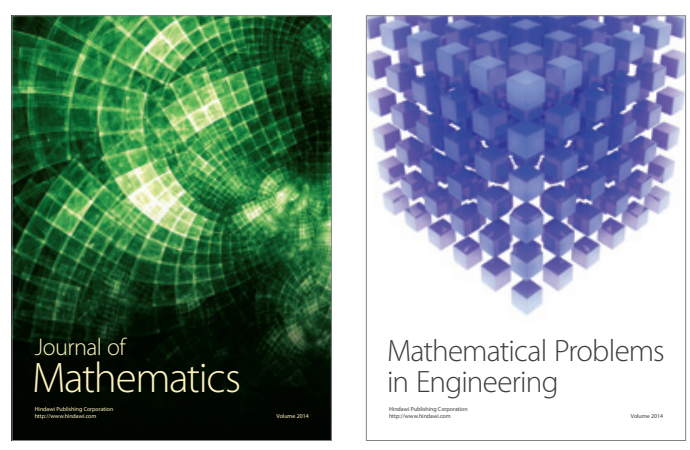

Mathematical Problems in Engineering
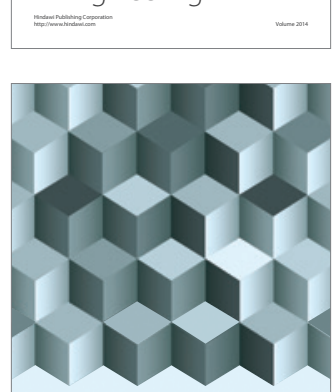

Journal of

Function Spaces
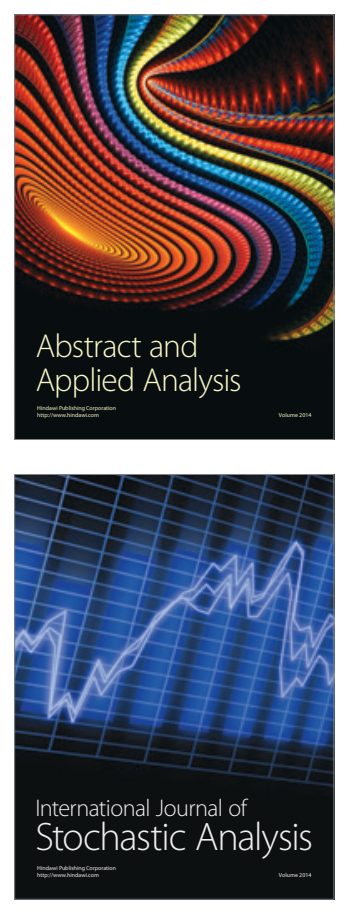

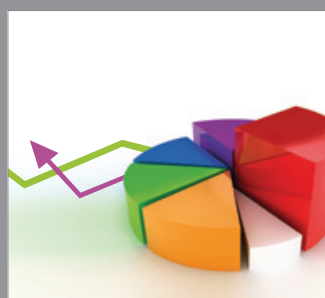

ournal of

Probability and Statistics

Promensencen
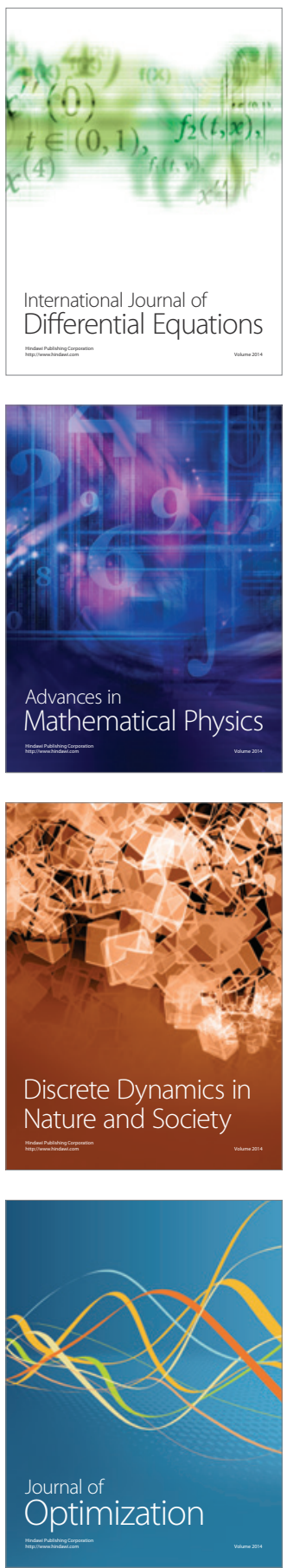